\section{Was soll die Leber im Gehirn?}

Forscher züchten Menschen-Minileber im Gehirn von Mäusen! Mit dieser Botschaft hat die Stammzellforschung kürzlich wieder einmal für Aufsehen gesorgt.

Da drängen sich einige Fragen auf: Was soll die menschliche Leber eigentlich im Mäusehirn? Kann sie dort ihre wichtigste Aufgabe, nämlich die Verstoffwechselung von Wein, Bier und Spirituosen überhaupt wahrnehmen? Trinken Mäuse wirklich soviel Alkohol, dass sie eine menschliche Leber brauchen? Und warum nur "mini“? Reicht das für einen

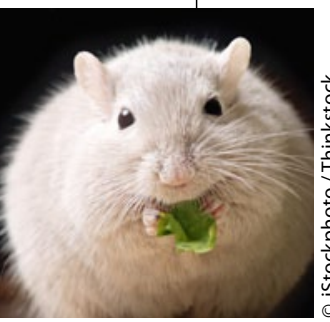

Mit menschlicher Minileber besser dran? durchschnittlichen Maustrinker? Oder soll diese Leber anơ schließend von der Maus in den Kopf des Menschen transferiert werden?

Warum eigentlich nicht? Im Kopf ist ja zumindest bei Menschen mit Alkoholproblemen oft viel Platz. Somit drängt sich dieser Hohlraum geradezu auf, wenn ein Plätzchen für eine neue Leber gesucht wird, weil die alte nicht mehr ausreichend funktioniert. Die kognitiven Funktionen könnten sich sogar verbessern, da das neue Organ Ammoniak direkt abfängt, bevor es das Restgehirn erreicht.

Wie die Sache bei den Mäusen weitergegangen ist, darüber gibt es keine Informationen. Hat sich ihr Trinkverhalten dem des Menschen angeglichen oder sind sie gar schlauer geworden? Hat die räumliche Nähe von Leber und Gehirn Vorteile, sodass wir sie auch beim Menschen anstreben sollten? Oder wäre ein Mini-Mausgehirn in der Leber vielleicht doch die bessere Alternative für den Menschen? Nach Meinung der Stammzellforscher eignet sich diese Technik nämlich grundsätzlich für alle Organe Peter Stiefelhagen .

Aktion gestartet:

\title{
Lauffeuer gegen COPD
}

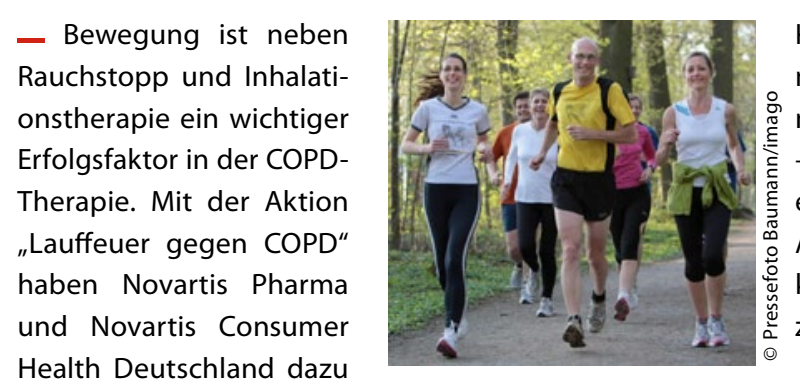
aufgerufen, ab dem 30. August gemeinsam in $80 \mathrm{Ta}$ gen die Welt zu umrunden. Jeder Teilnehmer kann ganz nach seinem Leistungsvermögen einen Beitrag zur Weltumrundung leisten, heißt es in einer Mitteilung zur jetzt gestarteten Aktion. Für jeden gelaufenen
Kilometer stellt Novartis gemeinnützigen Organisationen 50 Cent zur Verfügung - bis zu 20000 Euro können erlaufen werden.

Auf www.aktion-lauffeuer.de kann eine Schrittzähler-App zur messbaren Teilnahme am Lauf heruntergeladen und die Strecke mitverfolgt werden. Die App zählt die gelaufene Distanz des Nutzers und lädt sie auf die Lauffeuer-Website hoch. Alternativ kann unter der kostenfreien Telefonnummer 0800/2620109 ein Schrittzähler angefordert werden.

\section{Aktionswoche "Make Sense"}

\section{Aufklärung über Kopf-Hals-Tumoren}

— Vom 23.- 27. 9. 2013 findet die erste europäische Aufklärungswoche über Tumorerkrankungen im Kopf-Hals-Bereich statt. Ziel der von der European Head and Neck -Society (EHNS) initiierten Kampagne "Make Sense“ ist, das Bewusstsein für diese immer häufiger auftretenden Tumoren zu schärfen und durch Aufklärung über Früherkennungsmöglichkeiten die Heilungschancen zu verbessern (www.makesensecampaign. eu).

In Deutschland werden die Aktivitäten während der Aktionswoche u. a. von der Interdisziplinären Arbeitsgruppe Kopf-HalsTumoren der Deutschen Krebsgesellschaft (DKG), der Arbeitsgemeinschaft Onkologie der Deutschen Gesellschaft für Hals-NasenOhren-Heilkunde, dem Bundesverband der Kehlkopflosen und Kehlkopfoperierten e.V. sowie der Merck Serono GmbH, Darmstadt, der Boehringer Ingelheim Pharma $\mathrm{GmbH}$ \& Co. KG, Ingelheim, und der Transgene SA, Illkirch-Graffenstaden/Frankreich, unterstützt. Das ausführliche Programm deutschlandweiter Aktivitäten ist auf www. kopf-hals-krebs.de abrufbar.

Für den 24.9.2013 ist in der Zeit von 16:00-20:00 Uhr eine große Telefonaktion von Ärzten für Ärzte geplant: Experten der
Fachgebiete Hals-Nasen-Ohren-Heilkunde (0800/0005496), Radioonkologie (0800/ 0005497), Mund-Kiefer-Gesichts-Chirurgie (0800/0005498) und Onkologie (0800/ 0005499) beantworten unter den genannten Telefonnummern Fragen zu Kopf-HalsTumoren.

\section{Fürs Wartezimmer}

\section{Patientenbroschüre überaktive Blase}

Die Patientenbroschüre "20 Fragen 20 Antworten“ mit dem Titel „Wenn die Blase das Leben bestimmt " beantwortet die häufigsten Fragen, die im Zusammenhang mit Beschwerden bei überaktiver Blase auftreten.

Die Broschüre kann (im Paket mit jeweils 50 Exemplaren) kostenlos fürs Wartezimmer bestellt werden: vertrieb@springer.com oder per Fax an Nr. 06102/506240, Stichwort „20 Fragen zur überaktiven Blase“. 